\title{
A multi-level developmental approach towards understanding adolescent mental health and behaviour: rationale, design and methods of the LIFECOURSE study in Iceland
}

\author{
Thorhildur Halldorsdottir ${ }^{1}$ • Alfgeir Logi Kristjansson ${ }^{2} \cdot$ Bryndis Bjork Asgeirsdottir $^{1} \cdot$ Ingibjorg Eva Thorisdottir $^{1}$. \\ Jon Sigfusson ${ }^{3} \cdot$ Erla Maria Jonsdottir Tolgyes $^{3} \cdot$ Heiddis B. Valdimarsdottir ${ }^{1,4}$. John Allegrante ${ }^{5}$. \\ Inga Dora Sigfusdottir ${ }^{1,3,5}$
}

Received: 21 February 2020 / Accepted: 10 November 2020

(c) Springer-Verlag GmbH Germany, part of Springer Nature 2020

\begin{abstract}
Purpose Identifying and understanding modifiable risk and protective factors that can inform early detection and intervention to prevent adolescent emotional problems and harmful behaviours is among the most pressing modern-day public health challenges. This paper describes the rationale, objectives, methods, and anticipated outcomes of the LIFECOURSE study, a multi-level, bio-psychosocial prospective study designed to advance our understanding of factors that shape adolescent mental health and behaviour.

Methods Conducted by the Icelandic Centre for Social Research and Analysis at Reykjavik University, LIFECOURSE is a longitudinal population-based developmental study of Icelandic adolescents born in 2004. The study utilizes a comprehensive multi-informant assessment of individual, societal and biological factors measured across the lifespan. Data assembly and collection were conducted from 2016-2020 and utilize both retrospective and prospective data sources: (a) retrospective registry data assembled from seven national databases, (b) prospectively collected social surveys and (c) biomarker samples. Results Of the 3914 eligible adolescents, $60.8 \%(n=2378)$ provided informed parental consent and student assent to participate in the study, with approximately half of the participants being female $(n=1175,49.4 \%)$ and the majority being born in the capital area $(n=1455 ; 61.2 \%)$. The coverage of available data from the national databases and participation in the social surveys ranged from 81.7 to $100 \%$.

Conclusions Major gaps remain in our knowledge of how individual, societal and biological factors across the lifespan-from early life to adolescence-interact and shape the risk for emotional problems and harmful behaviours during adolescence. The LIFECOURSE study was designed to address this knowledge gap.
\end{abstract}

Keywords Adolescence $\cdot$ Bio-psychosocial model $\cdot$ Emotional problems $\cdot$ Delinquency $\cdot$ Stress $\cdot$ Substance abuse

Thorhildur Halldorsdottir thorhildurh@ru.is

$\bowtie$ Inga Dora Sigfusdottir ingadora@ru.is

1 Department of Psychology, Reykjavik University, Reykjavik, Iceland

2 Department of Social and Behavioral Sciences, West Virginia University, Morgantown, WV, USA

3 Icelandic Center for Social Research and Analysis, Reykjavik University, Reykjavik, Iceland

4 Population Health Science and Policy, Icahn School of Medicine At Mount Sinai, New York City, NY, USA

5 Department of Health and Behavior Studies, Teachers College, Columbia University, New York City, NY, USA

\section{Introduction}

Adolescence is a high-risk period for the onset of emotional problems and harmful behaviours $[1,2]$. Often these problems arise when young people experience major stress and vulnerabilities in their lives and/or are the result of being born into adverse circumstances [3-9]. Although, quite a lot is known about the effects of stress, there are still major gaps in our knowledge, especially in relation to how stress affects physiological and emotional reactions, and harmful behaviour, and whether those effects are preventable or reversible. Examples of early life stress include maternal illness during pregnancy, low birthweight, poverty and the experience of severe family conflict and violence. Common 
emotional problems and harmful behaviors that emerge during adolescence include anxiety, depressed mood, anger, substance use, delinquency, self-harm, and suicidal behaviors. These problems are associated with wide-ranging detrimental effects on the adolescent's well-being, which frequently persist into adulthood and may result in long-term morbidity and even premature mortality $[1,10]$. Alarmingly, recent studies have indicated that some of these large-scale problems may be on the rise [1,11-13]. As such, one of the most pressing modern-day challenges in public health is the identification of modifiable risk and protective factors for healthy adolescent development, which will inform more targeted early prevention and intervention strategies.

Currently, there is an emerging consensus that integrating factors at multiple biological and social levels is necessary to further our knowledge of human health and behaviour. For this, we need to move beyond a discipline-based approach [14]. Three main paradigms have been used to examine the effects of stress on mental health and behavior. The first stems from the literature on psychology and deals with the negative effects of stress on emotional disorders $[15,16]$. A second paradigm is rooted in sociology/criminology and studies the effects of what in sociology is called strain (stress) on delinquent (harmful) behavior [17]. Finally, the third paradigm explaining the effects of stress in peoples' lives, is physiological and uses the idea of allostatic load to capture the cumulative biological burden exacted on the body from repeated attempts to adapt to life's demands [18].

The LIFECOURSE project draws on these different theoretical frameworks and builds on prior work, including that of our own research team, conducted in Iceland to understand the potentially modifiable individual and social antecedents of adolescent harmful behaviours and whether and how positive environmental factors, such as social support, can benefit youth development [8, 19-23]. Through our research on determinants of substance use among Icelandic adolescents, we were able to formulate and implement an effective substance use prevention strategy that has demonstrated good success [24-26]. Although previous studies have been highly informative and sometimes effectively disseminated into practice, major gaps remain in our knowledge of how multiple-individual, societal and biological indicators from early on-interact and shape the risk for emotional problems and harmful behaviours in adolescence. Towards closing this knowledge gap, scholars have called for the increased use of a multi-level interdisciplinary developmental approach to advance our understanding of the biopsychosocial nature of risk and protective factors associated with mental health and behavioural outcomes in adolescents [14]. Thus, to provide the basis for a novel and comprehensive understanding of adolescent mental health and behavioural outomes, it is important to integrate individual, community and biological levels across the lifecourse. Such a holistic conceptual approach is necessary to disentangle the complex relationship between environmental and biological factors shaping adolescent development. Clearly, such findings put into practice would have important implications for policies for the mental health, education and criminal justice systems.

\section{Objectives}

In the LIFECOURSE study, we employ a multi-level, biopsychosocial approach to advance our understanding of risk and protective factors shaping mental health and behavioural outcomes among Icelandic adolescents. This entails a comprehensive multi-informant assessment of individual, societal and biological factors measured across the lifespan. Building and expanding on previous research, the primary objectives of the LIFECOURSE study are threefold (see Fig. 1 for the theoretical framework and hypothesized pathways):

\section{Objective 1}

To examine the links between high levels of early life stress and increased physiological stress (labelled "allostatic load" in the physiology literature), emotional reactions and harmful behaviour in adolescence.

\section{Objective 2}

To study the effects of stress in childhood, both on individual level and community level, on adolescent development.

\section{Objective 3}

To test whether high support in early adolescence may modify/buffer the effects of early life stress on physiological stress, emotional reactions, and harmful behaviour later in adolescence.

\section{Design and methods}

LIFECOURSE is a population-based developmental cohort study on Icelandic adolescents born in 2004 conducted by the Icelandic Centre for Social Research and Analysis (ICSRA) at Reykjavik University. Data assembling and collection was conducted during the years 2016 to 2020 . The study was reviewed and approved by the National Bioethics Committee of Iceland (11-078) and registered with the Personal Protection Authority. The study base comprised three retrospective and prospective data sources: (a) retrospective registry data assembled 


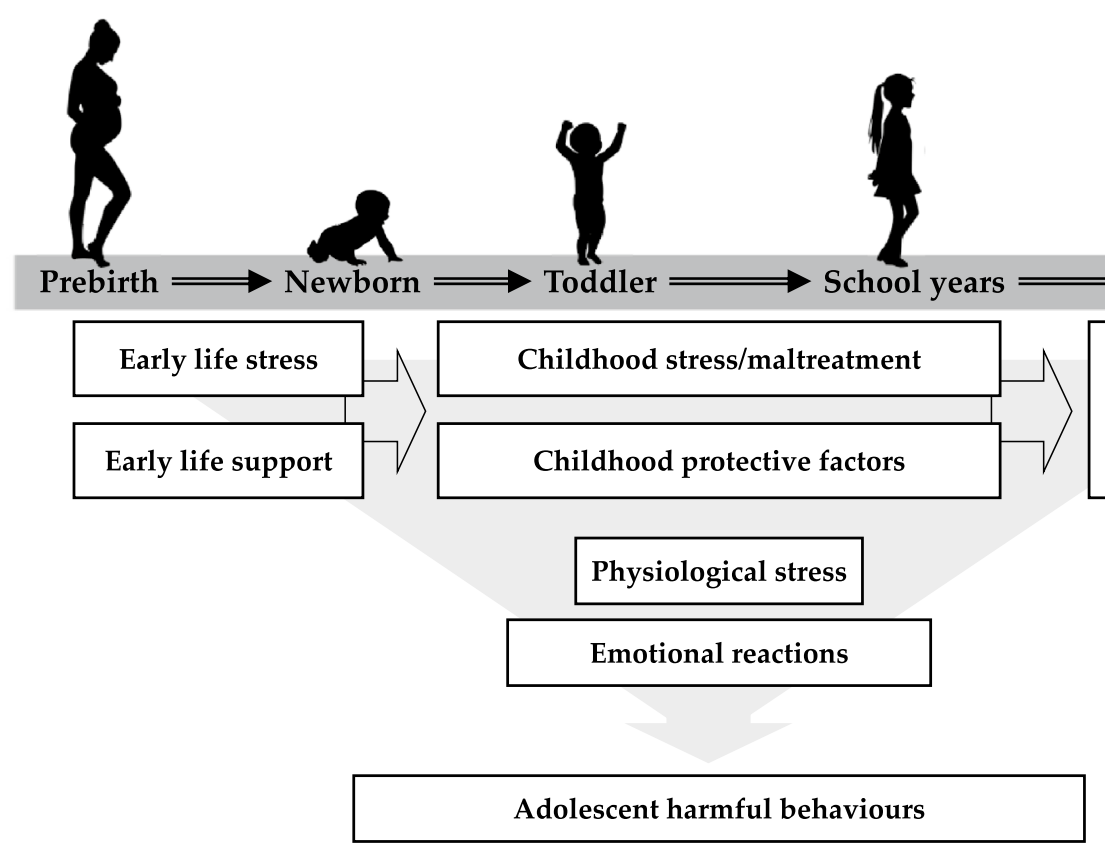

Fig. 1 The theoretical framework for which the LIFECOURSE study was conceived. In this simplified figure, we illustrate our model that provides a framework for investigating the effects of multiple envi-

from seven national databases, (b) prospectively collected social surveys and (c) biomarker samples. Figure 2 provides an overview of the enrollment and measurements in the LIFECOURSE study. Below, we provide a detailed ronmental factors experienced during specific developmental periods, and cumulatively over time, on physiological stress, emotional reactions, and harmful behaviour in adolescence

description of the setting and each data source, followed by details on the procedure and descriptive information for our pilot and main studies.

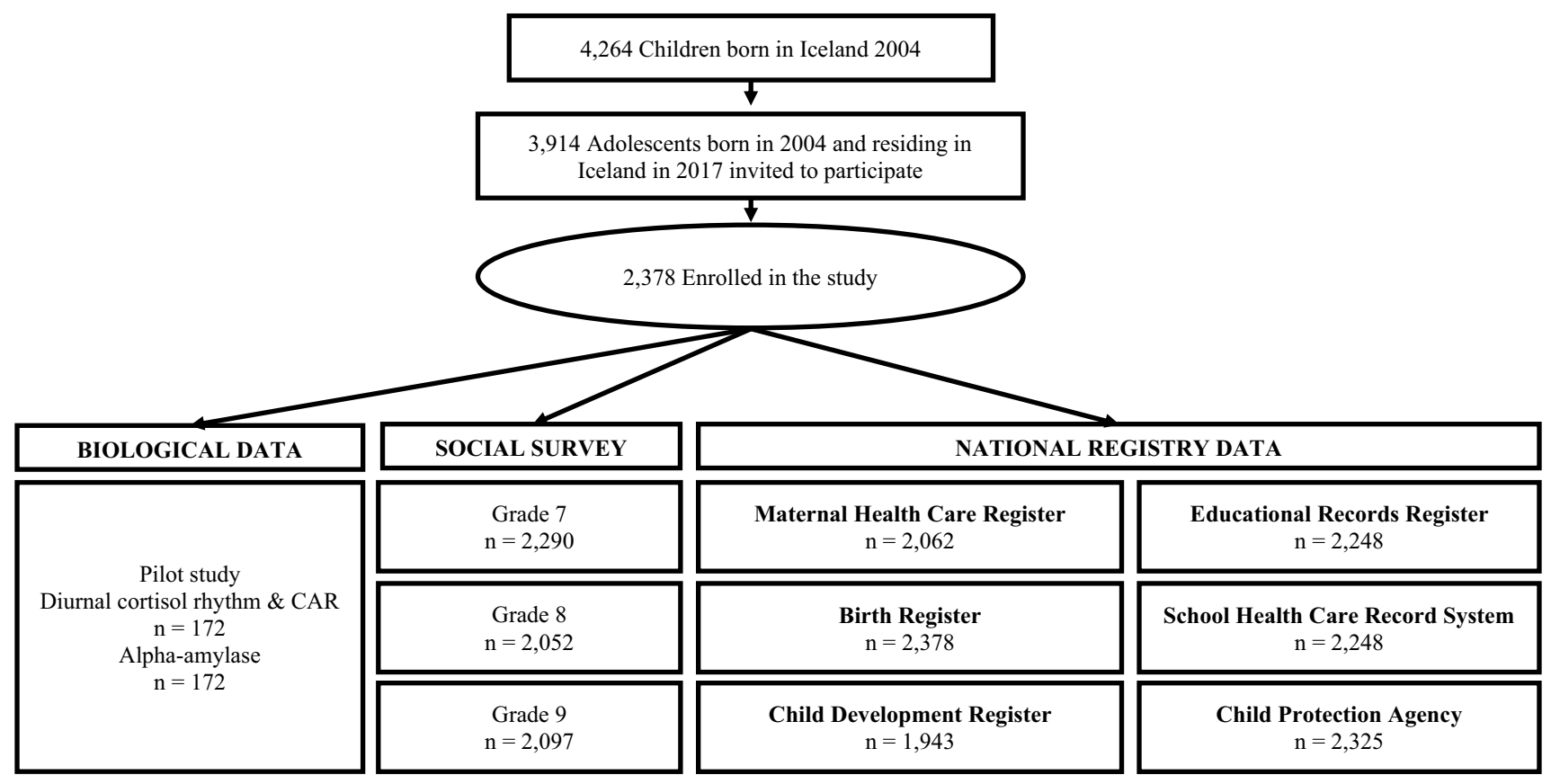

Fig. 2 Enrolment and measures in the LIFECOURSE study 


\section{Setting}

Iceland is a Nordic island nation populated by approximately 357,000 inhabitants, with approximately $62 \%$ of them residing in the capital, Reykjavik (based on information obtained 01.01 .2019 , [27]). Due to a combination of factors, the Icelandic research setting is uniquely well suited for applying a multi-level developmental framework to study the interaction between individual, societal and biological factors across the lifespan. First, the established infrastructure of the Icelandic health care system allow us to follow each child from pre-birth and onwards. A unique national identification number is issued to all children born in Iceland, Icelandic citizens born abroad and individuals with a legal permanent residence in Iceland. This number is used as the primary mode of identification in each of the national registry databases. Due to this individual-specific identification number, we are able to link demographic information, school-based trajectories, and various health outcomes across the lifespan, starting with maternal health care records in the prenatal period (see below for a detailed description). Second, the geographic isolation and homogeneity of the population enable the collection of ideal consecutive data on the whole population of children over time. Third, the Icelandic population is known for its positive attitude towards research participation (with up to $90 \%$ participation in our previous studies). Leveraging the combination of infastructure and population characteristics, we are able to create a unique multi-level description of emotional problems and harmful behaviors in adolescence on the individual and local community/neighbourhood levels.

\section{National registries}

Information on each participant was obtained from seven national registries: the National Maternal Health Care Registry, the National Birth Registry, the National Child Development Registry, National School Health Care Record System, the National Educational Records Registry, and the Child Protection Agency in the City of Reykjavik. Relevant databases were upgraded regularly throughout this study and other retrospective data may be added to the study in the future. Detailed information on the data extracted from each registry source is outlined below:

\section{National maternal health care register}

In Iceland, expectant mothers receive regular check-ups at their local healthcare centre at least seven times during pregnancy. These appointments occur around weeks 12, 16, and 20 of the pregnancy, and more frequently during the last 3 months of pregnancy. During these visits, familial demographic information, such as parental age, nationality, education, residency, co-habitation status and number of children, is registered. Familial risk factors for birth complications are also documented, e.g., family history of diabetes and multiparous pregnancies.

This database also includes maternal birth history and complications, including number of previous births, miscarriages, stillbirths, and number of premature births. Furthermore, information on mother's current physical health during (e.g., height, weight, blood pressure, gestational diabetes, hypertension/preeclampsia, smoking and medication status) and after the pregnancy (e.g., perineum, sleep after birth) is documented. Any ICD-10 diagnoses the mother meets criteria for are recorded. During these check-ups, health information on the fetus is also collected, including predicted weight, heart rate sound, and positioning.

\section{National birth register}

This database contains information on the family and child at the time of birth. Parental demographic information, such as parental co-habitation status, employment and residency is registered. This database also contains information on parental tobacco smoking and alcohol consumption during the pregnancy and established ICD-10 diagnoses of the mother. Information regarding the birth includes the location and time of birth, fetal positioning during birth, birth method and any interventions implemented during birth according to the NOMESCO Classification of Surgical Procedures (NCSP). Following birth, information regarding the newborn's health is documented, the infant's weight, length and head circumference at birth, APGAR scores and any ICD-10 diagnoses the newborn meets criteria for.

\section{National child development register}

In Iceland, families with newborns attend regular checkups at the local Health Care at the age of 6 weeks, 3 months, 6 months, 10 months, 18 months, 3.5 years old and 5 years. During the visits, a health care professional documents the child's height, weight and head circumference and completes a standardized developmental evaluation aimed at capturing critical aspects of the child's physical growth, fine motor and gross motor development, language comprehension, and social-emotional aspects of children's development. If the health care professional deems necessary based on the mother's presentation or history of depression, the Edinburgh Postnatal Depression Scale (EPDS; [28]) is administered to evaluate postpartum depressive symptoms. 


\section{School health care record system}

Health information is gathered by school nurses on children at the age of $6,9,12$ and 14 years. This data source includes information on the child's weight, height, immunizations, and documented psychiatric diagnoses. It also includes items capturing well-being (e.g., "How do you feel at school?") and life style indicators pertaining to eating habits (e.g., "What did you eat for breakfast during snack time at school?"), exercise (e.g., "Do you play outside often?"), cleanliness (e.g., "Did you brush your teeth this morning?"), sleeping habits ("When do you go to bed on school nights?"). Additionally, visits to the school nurse are documented throughout elementary school, which can be accumulated into a frequency measure of visits.

\section{Educational records register}

Icelandic students undergo mandatory standardized tests in Language Arts and Mathematics in 4th,7th and 9th grade (corresponding to ages 9,12 and 14 years old) to determine whether students are meeting the national curriculum proficiency objectives. The grades are normally distributed on a scale of 0-60, with an average score of 30 and a standard deviation of 10. Scores from these tests are documented by the Educational Testing Institute (ETI), which is overseen by the Ministry of Education, Science, and Culture.

\section{Child protection agency}

Referrals of childhood maltreatment and harmful behaviors are documented by the Child Protection Agency (CPA). These records include information of type of referral, number of reports, and tracking of the cases that receive further intervention. Referrals include the child being the victim of any type of neglect or abuse, child absenteeism from school, delinquency and other potentially harmful behaviors (e.g., substance use) during any time in their life. This data source also contains information on the child's diagnoses, family demographics (e.g., parental spousal status, financial status), parental information relevant to the referral (e.g., parental death, psychiatric disorders or history of abuse) and type of social services received.

\section{Prospective data collection}

In addition to the registry data, the LIFECOURSE study includes repeated annual self-report survey data, which are collected when participants are in grades 7, 8, and 9 (corresponding to ages 12,13, and 14 years old). Surveys were based on a standardized protocol that the ICSRA has used for over 20 years [29]. The social survey was designed to capture a broad picture of the adolescent's functioning.
Table 1 Social survey measures grade

\begin{tabular}{|c|c|c|c|}
\hline \multirow[t]{2}{*}{ Measures } & \multicolumn{3}{|c|}{ Grade } \\
\hline & 7 & 8 & 9 \\
\hline Demographic variables & + & + & + \\
\hline Relationship with parents and parenting practices & + & + & + \\
\hline Peer relationships and victimization & + & + & + \\
\hline Attitude towards school/learning & + & + & + \\
\hline Social media usage and bullying & + & + & + \\
\hline Leisure time activities & + & + & + \\
\hline Depressive and anxiety symptoms & + & + & + \\
\hline Self-harm and suicidal ideation or attempt & & + & + \\
\hline Anger and conduct problems & + & + & + \\
\hline Substance use (including nicotine) & + & + & + \\
\hline Diet and caffeine consumption & & + & + \\
\hline Weight and height & & + & + \\
\hline Trauma and stressful life events & + & + & + \\
\hline Self-concept & + & + & + \\
\hline Academic performance & + & + & + \\
\hline Learning difficulties & & + & + \\
\hline School absenteeism & + & + & + \\
\hline Social comparison & & + & + \\
\hline Neighborhood cohesion & & + & + \\
\hline Sexuality & & + & \\
\hline Employment & & + & + \\
\hline Porn viewing & & + & + \\
\hline
\end{tabular}

Table 1 provides an overview of the measures in the survey data by grade. Here, we highlight the measures directly relevant to our aforementioned study objectives rather than provide a detailed information on all of the measures in the survey. A more detailed list of measures is included on our website (www.lifecourse.is).

\section{Depressive symptoms, anxiety and anger}

Established measures were used to assess depressive symptoms, anxiety and anger over the past week. Namely, depressed mood (Cronbach $\alpha$ from 0.90-0.92), anxiety (Cronbach $\alpha$ from 0.79 to 0.81 ) and anger/hostility (Cronbach $\alpha$ from 0.82-0.84) were measured with the corresponding three out of nine subscales of the Symptom ChecklistRevised (SCL-90-R; [30, 31]). The Multidimensional Anxiety Scale for Children was used to measure symptoms of physical and social anxiety [32, 33] (Cronbach $\alpha$ from 0.88 to 0.92 and 0.89 to 0.92 , respectively).

\section{Delinquency}

Our measures of delinquency included the frequency of physical aggression (six items, e.g., "Have you punched someone?"), stealing (five items, e.g., "Have you broken into 
a building or car to steal something?"), bullying (six items, e.g., "Have you been part of a group that physically hurts someone?") within the past 12 months. Substance use was measured with items capturing the frequency of nicotine use (6 items, e.g., "How often have you smoked a cigarette?"), alcohol consumption ( 3 items, e.g. "How often have you been drunk?") and the use of illegal substances (16 items, e.g., "How often have you smoked marijuana?", "How often have you used amphetamine?").

\section{Self-harm and suicidal ideation and attempts}

The survey includes two items on the frequency of self-harm behaviors, irrespective of suicidal intent (e.g., "Have you had thoughts of harming yourself?", "Have you engaged in self-harm?"). Suicidal ideation among the adolescents was assessed with two items (i.e., "Have you ever thought of attempting suicide?"; "Have you told someone that you were thinking about attempting suicide?"). Suicidal attempts were also assessed with two items (i.e., "Have you ever attempted suicide?"). The prevalence of suicidal ideation/attempts within the adolescent's social network was also assessed with three items (e.g., "Has anyone told you that they are contemplating suicide?").

\section{Stressful and traumatic events}

The Negative Life Events Scale [34] was used to measure exposure to stressful (e.g., breakup with boyfriend/girlfriend) and traumatic events (e.g., victim of sexual abuse, death of a parent) within the past 30 days, 12 months, and more than 12 months ago (16 items). In addition to this scale, the survey contained items on being bullied (e.g., "How often in the past year have you been teased by a group of people?") and other types of potentially traumatic experiences (e.g., "Have you been coerced to send someone a photo of a sexual nature of yourself?").

\section{Family relationship and local community}

The adolescent-parent relationship was measured with 17 items on the time spent with parents, support received from parents (e.g., helping with homework) and parental social control and monitoring. We also included items on the adolescent's attitude towards the local community (e.g., "How much do you like living in your neighborhood?") and neighborhood cohesion (e.g., "My parents know many of our neighbors by name").

\section{Well-being and life style}

The survey also contained measures of well-being, such as the Short Warwick Edinburgh Mental Well-Being Scale
(SWEMWBS; [35], Cronbach $\alpha$ from 0.87 to 0.89), the Rosenberg self-esteem scale [36, 37] (Cronbach $\alpha$ from 0.89-0.91) and the body image subscale from the Offer Self-Image Questionnaire for Adolescents (Cronbach $\alpha$ from 0.76 to 0.83) [38, 39]. School well-being was measured with 16 items on the adolescent's attitude towards school (e.g., "Do you feel like you get enough help with your studies from your teachers?").

Participation and frequency of extracurricular (e.g., playing an instrument) and leisure activities (e.g., sport participation and other physical activity, playing video games), as well as social media usage (e.g., time spent on Facebook, Snapchat) were also assessed.

\section{Biological measures}

\section{Cortisol and alpha-amylase}

In 2018, we collected cortisol and alpha-amylase from a subset of the participants $(n=172)$. To obtain this subset, the schools with the highest participation rates were pooled together and we randomly selected schools until this pool until the participants reached 200 students. Next, we invited all the LIFECOURSE participants in these respective schools to participate in the saliva sampling. This methods was chosen over randomly selecting participants from the entire country given that school nurses were in charge of collecting the saliva samples. The participants in this subset provided saliva samples from passive drool at three time points: immediately after awakening, $30 \mathrm{~min}$ after waking up, and just before going to bed. This protocol assesses key elements of the basal cortisol rhythm (morning and evening levels, size of cortisol awakening response, slope of the diurnal cortisol rhythm across the day), and also provides measures of salivary alpha amylase (an indirect indicator of sympathetic activation) [40, 41]. During the saliva data collection, participants were asked to refrain from eating, drinking, smoking or vigorous exercise in the hour prior to each sample. Additionally, participants were asked about factors known to influence cortisol levels, such as smoking habits, caffeine intake, and use of psychotropic medication and oral contraceptives [40]. Participants also completed a sleep diary the morning of the saliva sampling in which they reported when they fell asleep and time of waking up and rated the quality of their sleep and measures on their current depressive and anxiety symptoms (as measured by the SCL-90).

\section{Procedure and data collection}

\section{Pilot study}

We conducted a pilot study to determine the quality and completeness of the data in the national registries. In the 
pilot study, we obtained registry data on all the children born in the year 2000 in Reykjavik, the capital of Iceland. The National University Hospital documented 1151 births (49.3\% females) in Reykjavik in 2000. For this pilot cohort, data from all the aforementioned national registries were assembled in years 2014-2016 but no prospective data were collected. The number of participants with available data for each registry was as follows: the National Maternal Health Care Register had records on 1,106 participants (96\%), the National Birth Register had records on 1149 individuals (99\%), the School Health Care Record System contained information for 6-, 9- and 12-year-olds for 1026, 1079 and 1115 participants, respectively $(89 \%, 94 \%$ and $97 \%)$, and the Reykjavik Child Protection Agency (CPA) contained information on 239 of the participants (100\%) which the agency had intervened with during the first 13 years of their lives. The successful completion of the pilot showed that obtaining sufficient information from national registries was feasible.

\section{Main study}

In the main study, all adolescents born in Iceland in the year 2004 and residing in Iceland in 2017 were invited to participate in the study. First, contact information for the sample was acquired through the National Statistical Bureau and sister agencies to obtain information on all children born in Iceland in 2004. A non-traceable, unique research identification number was created for each child to use across databases. A key that links individual names and contact information to research IDs is maintained by a third party at the Reykjavik City Primary Health Care Clinics and is not accessible to the research team.

Recruitment for the study and the social survey administration occurred in February in 2017, 2018 and 2019 in all upper secondary schools (corresponding to ages 12, 13 and 14 years old) in Iceland using procedures developed by ICSRA in collaboration with the Icelandic Ministry of Education, Science and Culture over a 20-year period [42]. For each school, an established contact person distributed an invitation letter to participate in the study to each student to take home for parental consent. Parents/caregivers were also contacted via email by each respective school authority. Teachers at individual school sites supervised the participation of students in the classroom and administered the survey questionnaire using a double-envelope system to identify students while distributing the surveys in classroom settings, omitting their identification post survey completion (non-traceable ID printed on each individual questionnaire for scanning and data processing). Students were instructed not to write their names, social security numbers, or any other identifying information anywhere on the questionnaire. The social survey material was all in Icelandic. Upon survey completion students were asked to place their completed questionnaire in a blank and pre-sealed envelope provided to them before returning it to the supervising teacher. For the participating youth, data from the social survey was combined with data from the national registries which was assembled in the years 2016-2019.

Figure 2 displays enrolment and the number of participants with available data from each dataset. In 2004, 4264 children were born in Iceland. Of those, 3914 adolescents were residing in Iceland in 2017 (91.8\%) and were invited to participate in the study. Of the 3914 adolescents, $60.8 \%$ $(n=2378)$ provided informed parental consent and student assent to participate in the LIFECOURSE study. Out of the 154 schools in Iceland with students in the 7th grade, 140 of them had students that enrolled in this study. Around half of the participants were female $(n=1175 ; 49.4 \%)$ and the majority were born in Reykjavik $(n=1455 ; 61.2 \%)$. Available national registry data on participants ranged from $81.7-100 \%$ and participation in the social surveys in grades 7,8 and 9 ranged from 86.3 to $96.3 \%$. Saliva to assess cortisol levels and alpha-amylase was obtained on 172 participants in 2018. Table 2 provides descriptives on selected demographic variables for the LIFECOURSE cohort across the lifespan. Preliminary findings suggest that the adolescents in the LIFECOURSE study are on par with same-aged peers in terms of gender $(\mathrm{OR}=0.942, p=0.366)$ and maternal age at birth $(\mathrm{OR}=1.001, p=0.167)$. However, compared to same-age peers, participants in the LIFECOURSE were more likely to have parents who are Icelandic citizens (mother: $\chi^{2}=49.90, p<0.001$, father: $\chi^{2}=79.41, p<0.001$ ) and live in a household where Icelandic is the primary spoken language $\left(\chi^{2}=53.25, p<0.001\right)$. Furthermore, youth with higher self-reported depressive and anxiety symptoms in 2017 were less likely to participate in $2018(p=0.004$ and $p=0.006$, respectively) but this association was not significant in 2019 ( $p=0.171$ and $p=0.121$, respectively). Higher levels of trauma exposure was also associated attrition $(p<0.001$ in 2018 and $p=0.008$ in 2019).

\section{Discussion}

The LIFECOURSE cohort was set up to shed light on the nuanced and complex relationship between bio-psychosocial indicators from early on, that shape adolescent outcomes. Building on accumulating evidence on the negative association of stressors and trauma exposure and poor health, our primary analyses at present center around early life stress and how it affects mental health and behavior during adolescence. Through these analyses, we expect to uncover novel modifiable targets to incorporate into prevention and treatment efforts. The large-scale, population-based design of this study ensures that our findings will be applicable to realworld clinical and public health decision-making. Building 
Table 2 Selected descriptives and characteristics of the LIFECOURSE cohort

Data source

\begin{tabular}{|c|c|c|c|c|}
\hline & & & & Data source \\
\hline \multicolumn{5}{|l|}{ Family demographics } \\
\hline Biological sex $N(\%)$ & & & & Birth registry \\
\hline Female & $1175(49.4 \%)$ & & & \\
\hline Male & $1201(50.5 \%)$ & & & \\
\hline Age at birth M (SD) & & & & Maternity registry \\
\hline Mother & $29.26(5.44)$ & & & \\
\hline Father & $31.95(6.18)$ & & & \\
\hline Icelandic citizenship $N(\%)$ & & & & Maternity registry \\
\hline Mother & $2324(97.73 \%)$ & & & \\
\hline Father & $1963(95.20 \%)$ & & & \\
\hline Parents cohabiting at birth $N(\%)$ & $2062(86.71 \%)$ & & & Birth registry \\
\hline Pregnancy & & & & Maternity registry \\
\hline Preeclampsia $N(\%)$ & $62(3.4 \%)$ & & & \\
\hline \multicolumn{5}{|l|}{ Maternal psychopathology ${ }^{+} N(\%)$} \\
\hline None endorsed & $1707(82.78 \%)$ & & & \\
\hline Endorsed & $262(12.71 \%)$ & & & \\
\hline Maternal smoking $N(\%)$ & $264(12.92 \%)$ & & & \\
\hline Birth & & & & Birth registry \\
\hline Weight in kilograms $M$ (SD) & $3.68(0.58)$ & & & \\
\hline Length in centimeters M (SD) & $51.69(2.58)$ & & & \\
\hline $\begin{array}{l}\text { Gestational age in weeks (ultrasound) } \\
\mathrm{M}(\mathrm{SD})\end{array}$ & $39.64(2.67)$ & & & \\
\hline \multirow[t]{2}{*}{ APGAR score at 5 min M (SD) } & $9.22(1.05)$ & & & \\
\hline & Time point & & & \\
\hline Toddlerhood & 6 months & 3.5 years & 5 years & Child development registry \\
\hline Weight in kilograms $M$ (SD) & $7.57(1.27)$ & $16.33(1.84)$ & $20.25(2.75)$ & \\
\hline Height in centimeters $\mathrm{M}$ (SD) & $67.33(4.1)$ & $100.86(3.76)$ & $112.79(4.58)$ & \\
\hline Childhood/Adolescence & 4th grade & 7th grade & 9th grade & Educational records registry \\
\hline Mathematics M (SD)* & $31.10(9.82)$ & $30.99(9.75)$ & $31.05(9.81)$ & \\
\hline Language Arts M (SD)* & $31.33(9.75)$ & $31.1(9.82)$ & $31.11(9.73)$ & \\
\hline Adolescence & 7th grade & 8th grade & 9th grade & Social surveys \\
\hline Depressive symptoms M (SD) & $13.54(6.99)$ & $14.26(8.26)$ & $14.91(8.57)$ & \\
\hline Physical anxiety symptoms M (SD) & $18.18(8.77)$ & $17.17(10.71)$ & $18.23(11.04)$ & \\
\hline Social anxiety M (SD) & $14.32(7.43)$ & $13.82(8.95)$ & $14.58(9.15)$ & \\
\hline Anger M (SD) & $9.88(6.52)$ & $7.04(4.33)$ & $7.15(4.30)$ & \\
\hline
\end{tabular}

Available data per participant differs as the information is merged from multiple databases in which the data was collected at different time points

+ Maternal psychopathology entails an endorsed history of depression, anxiety, alcohol abuse, drug abuse disorder or receiving pharmacotherapy

*The standardized scores from the Mathematics and Language Arts are normally distributed

on our highly successful ongoing collaboration with policy makers and community leaders at the municipality and government levels [42, 43], the knowledge generated from this cohort is expected to be disseminated into our researchbased prevention approach commonly referred to as Planet Youth [24, 26].

The data from the LIFECOURSE pilot study has generated research highlighting the long-lasting negative effect of various early life risk factors on academic outcomes during adolescence [44-46]. Briefly, one of these studies examined the relationship between multiple risk factors across the lifespan with academic achievement in 4th and 7 th grade [46]. The risk factors were derived from the national registry databases described above and included maternal smoking during pregnancy, parent's disability status, being born to a young mother ( $<19$ years old), number of children within 
the household, family income, frequency of visits to the school nurse, and reports of maltreatment. To summarize the results, there was a dose response effect between the risk factors and academic achievement, with exposure to a higher number of risk factors being associated with lower academic achievement in both 4th and 7th grade. Using latent growth curve modeling, we also found that maternal smoking during the first trimester of pregnancy was associated with lower standardized score in mathematics and language arts in 4th grade and this negative association persisted across 4th to 10 th grade $[44,45]$. This association remained significant after controlling for gender, income, parental cohabitation, and baseline academic performance.

Importantly, our recent work indicates that findings from the LIFECOURSE study may be generalizable not only to Icelandic youth but also adolescents worldwide. Specifically, the drastic decrease in adolescent substance use following the implementation of the prevention approach has received worldwide attention from researchers and policy makers alike and spurred on many collaborations with countries aspiring to do the same. At present, the implementation of the Planet Youth approach has begun or is underway in 31 countries worldwide [26, 43, 47]. Preliminary findings of the dissemination to other countries are promising, albeit it is early days when it comes to measuring largescale societal changes. We anticipate future findings from the LIFECOURSE study to further benefit and strengthen these efforts.

\section{Strengths and limitations}

The LIFECOURSE study has several important strengths. First, the study leverages a multi-level developmental approach in which a wide range of retrospective and prospective multi-informant data (e.g., health care records, maternal- and self-report) across the lifespan has been collected. The study contains broad indicators of the global functioning, ranging from mental and physical health outcomes to academic performance, for the majority of Icelandic adolescents born in 2004. This makes it possible to study the combined environmental - at an individual and societal level-and biological effects at varying developmental stages on adolescent mental health and beyond. Second, attrition and missing data in the national registries and social survey data were minimal compared to most longitudinal studies. This is due to the detailed documentation within the health system and positive attitude towards research in Iceland. Third, through the unique Icelandic national registries, we are able to follow-up on the participants' health outcomes into adulthood because all medical visits are recorded in the registries.

Several limitations should also be noted. First, the adolescents participating in the study differed somewhat from the non-participating same-aged peers on parental education, employment status and citizenship. This is to be expected given the bias that families who are better off tend to participate in school-based studies [48] and all the material completed by the adolescent (e.g., assent and social surveys) or the caregiver (e.g., consent) was in Icelandic. As such, caution will need to be exercised when interpreting the findings, as they may not generalize to the entire population, especially expatriates in Iceland. However, previous research by our group has shown that variable relationships pertaining to risk and protective factors for substance use and delinquency reveal that youth in Iceland are no different from youth in other Western countries [49-52].

Taken together, the LIFECOURSE study is exceptionally placed to advance our understanding on how environmental (at the individual and societal level) and biological factors across the lifespan shape adolescent mental health and behavior.

\section{Collaboration}

The LIFECOURSE study has an open policy in terms of collaborating with other research groups. For interested researchers, collaboration requests should be addressed to the study's Princial Investigator, Prof. Inga Dora Sigfusdottir(ingadora@ru.is).The proposed study aims and potential overlap of requests with ongoing studies are discussed with the LIFECOURSE team. Once approved, a LIFECOURSE team member will be assigned to supervise and assist with the project.

Acknowledgements The authors thank all youths participating in this epidemiological study, the schools that made the study possible with their help in terms of data collection and the research assistants that assisted with the data collection.

Author contributions IDS conceived and designed the LIFECOURSE study in collaboration with ALK, BBA, JA, and HBV. Data collection was carried out by JS, EMJT, and IET. TH wrote the first draft of the manuscript, managed data cleaning and ran all analyses. All authors contributed to the final version of the manuscript.

Funding The social survey data collection, compilation of the registry data, as well as the cortisol and alpha amylase measurements, of the LIFECOURSE study were funded by a Project Grant (20658021-22-23) from RANNIS, the Icelandic Centre for Research and a Research Consolidator Grant from the European Research Council (ERC-CoG-2014-647860). The ERC had no role in formulating the study objectives and did not impact the analyses, reporting or selection of a journal outlet.

Availability of data and material The study has an open policy in terms of collaborating with other research groups. For interested researchers, collaboration requests should be addressed to the study's Princial Investigator, Prof. Inga Dora Sigfusdottir (ingadora@ru.is). The proposed study aims and potential overlap of requests with ongoing studies are discussed with the LIFECOURSE team. Once approved, a 
LIFECOURSE team member will be assigned to supervise and assist with the project.

\section{Compliance with ethical standards}

Conflicts of interest The authors have no conflicts of interest to declare.

Ethical approval All procedures performed in studies involving human participants were in accordance with the ethical standards of the institutional and/or national research committee and with the 1964 Helsinki Declaration and its later amendments or comparable ethical standards. The study was approved by the National Bioethics Committee of Iceland (11-078) and registered with the Personal Protection Authority.

Consent to participate Informed consent from the parents and assent from the adolescents was obtained.

Consent for publication No potentially identifying information is included in this manuscript.

Code availability Not applicable for this paper.

\section{References}

1. Kassebaum N, Kyu HH, Zoeckler L, Olsen HE, Thomas K, Pinho C, Bhutta ZA, Dandona L, Ferrari A, Ghiwot TT (2017) Child and adolescent health from 1990 to 2015: findings from the global burden of diseases, injuries, and risk factors 2015 study. JAMA Ped 171(6):573-592

2. Paus T, Keshavan M, Giedd JN (2008) Why do many psychiatric disorders emerge during adolescence? Nat Rev Neurosci 9(12):947-957

3. Aseltine RH Jr, Gore S, Gordon J (2000) Life stress, anger and anxiety, and delinquency: an empirical test of general strain theory. J Health Soc Behav 2000:256-275

4. Fairbrother G, Stuber J, Galea S, Fleischman AR, Pfefferbaum B (2003) Posttraumatic stress reactions in New York City children after the September 11, 2001, terrorist attacks. Ambul Pediatr 3(6):304-311

5. Goodyer IM (1990) Life experiences, development, and childhood psychopathology. John Wiley and Sons, New York

6. Pfefferbaum B, Stuber J, Galea S, Fairbrother G (2006) Panic reactions to terrorist attacks and probable posttraumatic stress disorder in adolescents. J Traum Stress Off Pub Internat Soc Traum Studies 19(2):217-228

7. Santelli JS, Kaiser J, Hirsch L, Radosh A, Simkin L, Middlestadt S (2004) Initiation of sexual intercourse among middle school adolescents: the influence of psychosocial factors. J Adolesc Health 34(3):200-208

8. Sigfusdottir I-D, Farkas G, Silver E (2004) The role of depressed mood and anger in the relationship between family conflict and delinquent behavior. J Youth Adolesc 33(6):509-522

9. Bao W-N, Whitbeck LB (2000) Hoyt DR (2000) Abuse, support, and depression among homeless and runaway adolescents. J Health Soc Behav 1:408-420

10. Kieling C, Baker-Henningham H, Belfer M, Conti G, Ertem I, Omigbodun O, Rohde LA, Srinath S, Ulkuer N, Rahman A (2011) Child and adolescent mental health worldwide: evidence for action. Lancet 378(9801):1515-1525

11. Collishaw S (2015) Annual research review: secular trends in child and adolescent mental health. JCPP 56(3):370-393
12. Morgan C, Webb RT, Carr MJ, Kontopantelis E, Green J, ChewGraham CA, Kapur N, Ashcroft DM (2017) Incidence, clinical management, and mortality risk following self harm among children and adolescents: cohort study in primary care. BMJ 359:4351

13. Trivers KF, Phillips E, Gentzke AS, Tynan MA, Neff LJ (2018) Prevalence of cannabis use in electronic cigarettes among US youth. JAMA Ped 172(11):1097-1099

14. Sigfusdottir ID, Kristjansson AL, Thorlindsson T, Allegrante JP (2016) Stress and adolescent well-being: the need for an interdisciplinary framework. Health Promot Int 32(6):1081-1090

15. Pearlin LI (1999) Stress and mental health: A conceptual overview. In: Horwitz AV, Scheid TL (eds) A handbook for the study of mental health: social contexts, theories, and systems. Cambridge University Press, pp 161-175

16. Thoits PA (2011) Mechanisms linking social ties and support to physical and mental health. J Health Soc Behav 52(2):145-161

17. Agnew R (1992) Foundation for a general strain theory of crime and delinquency. Criminology 30(1):47-88

18. Danese A, McEwen BS (2012) Adverse childhood experiences, allostasis, allostatic load, and age-related disease. Physiol Behav 106(1):29-39

19. Kristjansson AL, Sigfusdottir ID, Allegrante JP, Helgason AR (2008) Social correlates of cigarette smoking among Icelandic adolescents: a population-based cross-sectional study. BMC Public Health 8(1):86

20. Kristjansson AL, James JE, Allegrante JP, Sigfusdottir ID, Helgason AR (2010) Adolescent substance use, parental monitoring, and leisure-time activities: 12-year outcomes of primary prevention in Iceland. Prev Med 51(2):168-171

21. Sigfusdottir ID, Kristjansson AL, Thorlindsson T, Allegrante JP (2008) Trends in prevalence of substance use among Icelandic adolescents, 1995-2006. Sub Abuse Treat Prev Policy 3(1):12

22. Sigfúsdóttir ID, Thorlindsson T, Kristjánsson ÁL, Roe KM, Allegrante JP (2009) Substance use prevention for adolescents: the Icelandic model. Health Promot Internat 24(1):16-25

23. Sigfusdottir ID, Asgeirsdottir BB, Sigurdsson JF, Gudjonsson GH (2011) Physical activity buffers the effects of family conflict on depressed mood: a study on adolescent girls and boys. J Adoles 34(5):895-902

24. Kristjansson AL, Mann MJ, Sigfusson J, Thorisdottir IE, Allegrante JP, Sigfusdottir ID (2020a) Development and guiding principles of the icelandic model for preventing adolescent substance use. Health Promot Pract 21(1):62-69

25. Kristjansson AL, Mann MJ, Sigfusson J, Thorisdottir IE, Allegrante JP, Sigfusdottir ID (2020b) Implementing the icelandic model for preventing adolescent substance use. Health Promot Practice 21(1):70-79

26. Sigfusdottir ID, Soriano HE, Mann MJ, Kristjansson AL (2020) Prevention is possible: a brief history of the origin and dissemination of the Icelandic prevention model. Health Promot Pract 21(1):58-61

27. Inhabitants in Iceland - Overview [Internet]. Statistics Iceland [Hagstofa Íslands]; 2019 Jan [cited 2019 Dec 16]. Available from: https://statice.is/statistics/population/inhabitants/overview/. Accessed 16 Dec 2019

28. Cox JL, Holden JM, Sagovsky R (1987) Detection of postnatal depression: development of the 10-item edinburgh postnatal depression scale. BJPsych 150(6):782-786

29. Kristjansson AL, Sigfusson J, Sigfusdottir ID, Allegrante JP (2013) Data collection procedures for school-based surveys among adolescents: the Youth in Europe Study. J Sch Health 83(9):662-667

30. Derogatis L, Lipman R, Covi L (1975) Symptom checklist SCL90-R. Clin Psych Res Baltimore 2:7 
31. Rytilä-Manninen M, Fröjd S, Haravuori H, Lindberg N, Marttunen M, Kettunen K, Therman S (2016) Psychometric properties of the symptom checklist-90 in adolescent psychiatric inpatients and age-and gender-matched community youth. J Child Adol Psyc Mental Health 10(1):23

32. March JS, Parker JD, Sullivan K, Stallings P, Conners CK (1997) The multidimensional anxiety scale for children (MASC): factor structure, reliability, and validity. JACAAP 36(4):554-565

33. Thor Olason D, Blöndahl Sighvatsson M, Smari J (2004) Psychometric properties of the multidimensional anxiety scale for children (MASC) among Icelandic schoolchildren. Scand J Psychol 45(5):429-436

34. Wills TA, McNamara G, Vaccaro D, Hirky AE (1997) Escalated substance use: a longitudinal grouping analysis from early to middle adolescence. In: Marlatt GA, VandenBos GR (eds) Addictive behaviors: readings on etiology, prevention, and treatment. American Psychological Association, pp 97-128

35. Stewart-Brown S, Tennant A, Tennant R, Platt S, Parkinson J, Weich S (2009) Internal construct validity of the Warwick-Edinburgh mental well-being scale (WEMWBS): a Rasch analysis using data from the Scottish health education population survey. Health Quality Life Outcomes 7(1):15

36. Rosenberg M (1965) Rosenberg self-esteem scale (SES). Soc Adolesc Self-image 1:7

37. Asgeirsdottir BB, Gudjonsson GH, Sigurdsson JF, Sigfusdottir ID (2010) Protective processes for depressed mood and anger among sexually abused adolescents: the importance of self-esteem. Personality Individ Differ 49(5):402-407

38. Offer D, Howard KI (1972) An empirical analysis of the offer self-image questionnaire for adolescents. Arch Gen Psychiatry 27(4):529-533

39. Asgeirsdottir BB, Ingolfsdottir G, Sigfusdottir ID (2012) Body image trends among Icelandic adolescents: a cross-sectional national study from 1997 to 2010. Body image 9(3):404-408

40. Adam EK, Kumari M (2009) Assessing salivary cortisol in largescale, epidemiological research. Psychoneuroendocrinology 34(10):1423-1436

41. Granger DA, Kivlighan KT, Fortunato C, Harmon AG, Hibel LC, Schwartz EB, Whembolua G-L (2007) Integration of salivary biomarkers into developmental and behaviorally-oriented research: problems and solutions for collecting specimens. Physiol Behav 92(4):583-590

42. Kristjansson AL, Mann MJ, Sigfusson J, Thorisdottir IE, Allegrante JP, Sigfusdottir ID (2019) Development and guiding principles of the Icelandic model for preventing adolescent substance use. Health Promot Pract 1:5248399

43. Asgeirsdottir BB, Kristjansson AL, Sigfusson J, Allegrante JP, Sigfusdottir ID (2020) Trends in substance use and primary prevention variables among adolescents in Lithuania, 2006-19. European J Pub Health. https://doi.org/10.1093/eurpub/ckaa097

44. Kristjansson AL, Thomas S, Lilly CL, Thorisdottir IE, Allegrante JP, Sigfusdottir ID (2018) Maternal smoking during pregnancy and academic achievement of offspring over time: a registry databased cohort study. Prev Med 113:74-79

45. Kristjansson AL, Thorisdottir IE, Steingrimsdottir T, Allegrante JP, Lilly CL, Sigfusdottir ID (2017) Maternal smoking during pregnancy and scholastic achievement in childhood: evidence from the LIFECOURSE cohort study. Euro J Public Health 27(5):850-855

46. Ragnarsdottir LD, Kristjansson AL, Thorisdottir IE, Allegrante JP, Valdimarsdottir H, Gestsdottir S, Sigfusdottir ID (2017) Cumulative risk over the early life course and its relation to academic achievement in childhood and early adolescence. Prev Med 96:36-41

47. Hoare E, Thorisdóttir IE, Kristjansson AL, Sigfusdóttir ID, Hayward J, Allender S, Strugnell C, Reavley N, Patton G, Berk M (2019) Lessons from Iceland: Developing scalable and sustainable community approaches for the prevention of mental disorders in young Australians. Mental Health Prev 15:200166

48. Anderman C, Cheadle A, Curry S, Diehr P, Shultz L, Wagner E (1995) Selection bias related to parental consent in school-based survey research. Eval Rev 19(6):663-674

49. Kristjansson AL, Sigfusdottir ID, Thorlindsson T, Mann MJ, Sigfusson J, Allegrante JP (2016) Population trends in smoking, alcohol use and primary prevention variables among adolescents in Iceland, 1997-2014. Addiction 111(4):645-652

50. Kristjansson AL, Sigfusdottir ID, James JE, Allegrante JP, Helgason AR (2010) Perceived parental reactions and peer respect as predictors of adolescent cigarette smoking and alcohol use. Addict Behav 35(3):256-259

51. Sigfusdottir ID, Kristjansson AL, Agnew R (2012) A comparative analysis of general strain theory. J Criminal Just 40(2):117-127

52. Kristjansson AL, Sigfusdottir ID, Allegrante JP (2013) Adolescent substance use and peer use: a multilevel analysis of cross-sectional population data. Subst Abuse Treat Prev Policy 8:27 